\title{
Sliding states of a soft-colloid cluster crystal: Cluster versus single-particle hopping
}

\author{
Mirko Rossini, Lorenzo Consonni, Andrea Stenco, Luciano Reatto, and Nicola Manini* \\ Dipartimento di Fisica, Università degli Studi di Milano, via Celoria 16, 20133 Milano, Italy
}

(Received 30 March 2018; revised manuscript received 9 May 2018; published 22 May 2018)

\begin{abstract}
We study a two-dimensional model for interacting colloidal particles which displays spontaneous clustering. Within this model we investigate the competition between the pinning to a periodic corrugation potential and a sideways constant pulling force which would promote a sliding state. For a few sample particle densities and amplitudes of the periodic corrugation potential we investigate the depinning from the statically pinned to the dynamically sliding regime. This sliding state exhibits the competition between a dynamics where entire clusters are pulled from a minimum to the next and a dynamics where single colloids or smaller groups leave a cluster and move across the corrugation energy barrier to join the next cluster downstream in the force direction. Both kinds of sliding states can occur either coherently across the entire sample or asynchronously: the two regimes result in different average mobilities. Finite temperature tends to destroy separate sliding regimes, generating a smoother dependence of the mobility on the driving force.
\end{abstract}

DOI: 10.1103/PhysRevE.97.052614

\section{INTRODUCTION}

The standard atomistic approach to tribology, i.e., the study on friction dissipation and wear, usually focuses on indestructible "crushproof" particles, typically atoms or molecules [1-9]. Recent works [10-14] have brought colloids into the realm of tribology, by letting repulsive hard-core particles interact with a periodic "corrugation potential," generated by means of optical forces and driven by viscous drag. The conditions of that experiment match the single indestructible particle paradigm. Colloids and, more generally, soft matter systems would, however, allow one to investigate situations where complex objects, carrying an internal structure, under the stress produced by external interactions and driving, can alter their internal structure both in shape and even in the number of the component subparticles. Thus, one can expect the presence of a varied set of regimes of response of the system to the external perturbation. In the present work, we address precisely this last situation by means of molecular-dynamics (MD) simulations of a system forming microphases. Microphase formation can take place under a number of conditions on the interparticle interactions and on the state variables in three dimensions [15-19] as well as in two dimensions [20-23]. The microphases can be disordered $[15,18,21,22]$ as well as ordered in a crystallinelike state or other patterned structures, like stripes and lamellae [17,21-25], or even as an ordered bicontinous state [26] in which both components of a two-component system span the space. Examples of microphase forming systems are block polymers [24,25] or hard colloids with competing interactions, e.g., short-range attraction-long-range repulsion, like for some colloid-polymer mixtures [15,16,18,20-22]. Certain soft-matter systems, like star polymers or dendrimers, in a good solvent can interpenetrate each other to a large extent so that the effective interaction between the centers of mass of two

\footnotetext{
*nicola.manini@fisica.unimi.it
}

such entities has a soft-core character. Also such systems can form microphases, in which the colloids spontaneously aggregate into clusters, and the clusters are organized in a crystalline state [27,28]. Rheological properties of cluster fluid phase [29] and of cluster crystal phase [30] have been investigated, but no study has yet addressed the tribology of such systems.

In the present work, we simulate a system of interacting colloidal particles whose mutual interaction potential generates the spontaneous formation of clusters. In particular, we concentrate on a 2D geometry with repulsive interactions, like in many experiments carried out with colloids [10,31-35], but the concepts introduced here may be relevant for vortexes in superconductors [36] too. The novelty compared to these experiments is the adoption of an interparticle interaction which supports the spontaneous formation of cluster phases. Cluster dynamics has been investigated in the past [37-39]: in these works clustering was forced by an external potential, while in the present work clustering is the intrinsic result of the particle-particle interaction and is retained even when any external potentials are turned off.

Section II introduces and motivates the model. As a first step, in Sec. III we characterize the $T=0$ equilibrium states of the model free from external interactions. We study the ground-state energy of several regular arrangements of clusters formed by $n$ particles (with $n$ from 1 to 6 , that we will refer to as $n$ clusters) as a function of the mean colloids density, obtaining a $T=0$ phase diagram. Then Sec. IV reports the investigation of the dynamics of the resulting arrays of clusters interacting with a lattice-matched external periodic potential and an homogeneous pulling force. Specifically, we report the mobility curves for these cluster systems, as a function of the intensity of the pulling force, for a few values of the amplitude of the corrugation potential. We characterize in detail the different sliding regimes, with entire clusters advancing, or only parts of them. The main outcome of this research is that the clusters internal dynamics is indeed affecting significantly the depinning force and the overall mobility. 


\section{THE MODEL}

\section{A. The interparticle interaction}

Spontaneous cluster formation can be the result of competitive interactions $[15,16,18,20-22]$, but clusters can also form due to soft potentials $[23,27,28]$. Here we adopt an interaction of the latter type. We consider the following form for the pairwise interaction potential energy:

$$
\phi_{\mathrm{cc}}(r)=\frac{A_{0}}{r^{6}+R_{c}^{6}}+\frac{B_{0}}{r^{6}} .
$$

This interaction, depicted in Fig. 1, consists of two terms: a soft-core repulsive interaction and a weak hard-core repulsion. The $B_{0}=0$ (purely soft-core) model has been fully characterized in two dimensions in Ref. [23]: its phase diagram consists in a high-temperature low-density regular fluid phase and a low-temperature high-density cluster phase. The reason for the cluster phase is that when the interparticle distance decreases under $r \lesssim R_{c}$, the potential energy $\phi_{\mathrm{cc}}(r)$ flattens out to a nearly constant energy, with the result of producing a quite small repulsive force. As a result, in one dimension a particle in between two other particles kept at fixed positions, when the distance between these fixed particles is not too large, may be better off energetically by coming closer to either of them, rather than remaining at the middle point. This suggests the mechanism whereby, in any dimensionality and at large enough density, a spontaneous symmetry breaking leading to clustering can be energetically favorable against a uniform fluid phase. A detailed mathematical criterion has been developed for the formation of cluster phases in the case of soft-core potentials in terms of their Fourier transform becoming negative in a range of finite wave vector [40]. At high $T$, entropic effects and fluctuations tend to favor the uniform fluid phase against cluster formation. In the opposite $T \rightarrow 0$ extreme, for $B_{0}=0$ no mechanism keeps the colloids apart, and so the clustering tendency would reach the extreme limit of collapsed pointlike clusters with all colloids of a cluster sitting

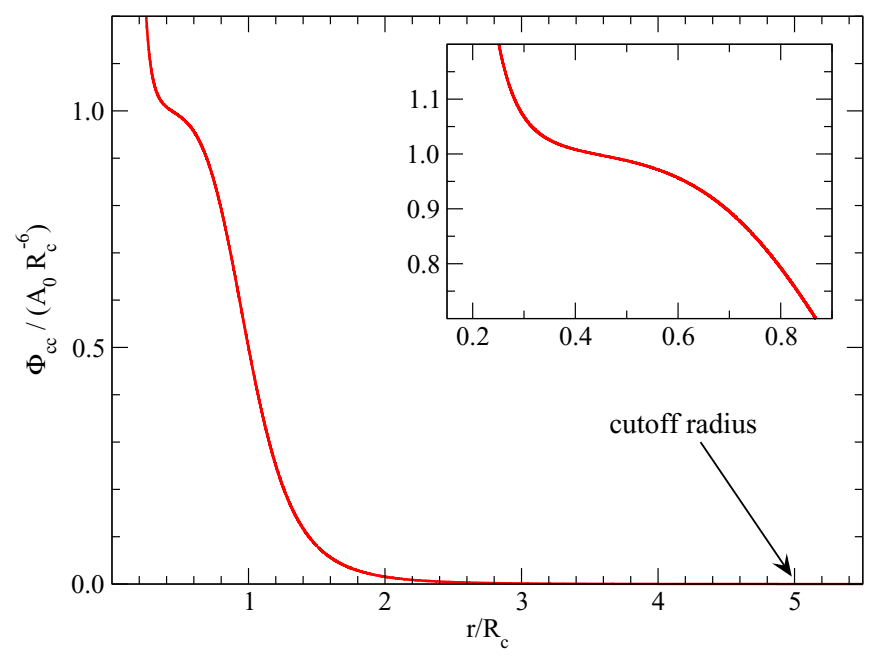

FIG. 1. The adopted colloid-colloid potential $\phi_{\mathrm{cc}}(r)$, Eq. (1), consisting on a soft-core function plus a weak short-range hard-core term that we introduced to prevent the collapse of clusters at $T=0$. Inset: detail of the short-distance plateau.
TABLE I. The system of units adopted in the simulations. Every physical quantity is expressed in terms of these natural model units.

\begin{tabular}{lc}
\hline \hline Physical quantity & System units \\
\hline Length & $R_{c}$ \\
Energy & $E_{0}=A_{0} / R_{c}^{6}$ \\
Mass & $m$ \\
Number density & $R_{c}^{-2}$ \\
Force & $F_{0}=A_{0} / R_{c}^{7}$ \\
Time & $t_{0}=R_{c}^{4} \sqrt{m / A_{0}}$ \\
Velocity & $v_{0}=R_{c}^{-3} \sqrt{A_{0} / m}$ \\
Viscous damping & $\eta_{0}=R_{c}^{-4} \sqrt{A_{0} m}$ \\
Mobility & $\mu_{0}=R_{c}^{4} / \sqrt{A_{0} m}$ \\
\hline \hline
\end{tabular}

exactly at the same position. To prevent this singular behavior we add the hard-core $B_{0}$ term of Eq. (1) for whose coefficient we adopt a relatively small value $B_{0}=5 \times 10^{-5} A_{0}$ [41] to perturb the phase diagram as little as possible, at least in the not-too-high-density region that we are interested in.

We take the repulsive-potential characteristic distance $R_{c}$, the characteristic energy $A_{0} / R_{c}^{6}$, and the mass $m$ of the colloidal particles as fundamental units. In the following we express all physical quantities in terms of suitable model units, as listed in Table I.

\section{B. The equations of motion}

In simulations, we let the entire system evolve following the standard Langevin dynamics in two dimensions [42], under the effect of an external periodic corrugation potential $U_{\text {ext }}(\mathbf{r})$ and a constant driving force $F$ applied to each particle. The equation of motion for the $j$ th particle is

$$
\begin{aligned}
m \ddot{\mathbf{r}}_{j}= & F \hat{\mathbf{x}}-\eta \dot{\mathbf{r}}_{j}+ \\
& -\nabla_{\mathbf{r}_{j}}\left[\sum_{k \neq j}^{N} \phi_{\mathrm{cc}}\left(\left|\mathbf{r}_{k}-\mathbf{r}_{j}\right|\right)+U_{\mathrm{ext}}\left(\mathbf{r}_{j}\right)\right]+\xi_{j} .
\end{aligned}
$$

Here $\eta$ is the coefficient of viscous friction associated to the fluid where the colloids move; we generate an overdamped dynamics by adopting a large $\eta=28 \eta_{0}$. The $\xi_{j}$ terms are Gaussian-distributed random forces with amplitude $\sigma=\sqrt{2 m \eta k_{\mathrm{B}} T / \Delta t}$, where $\Delta t$ is the simulation time step: they simulate the collisions of the molecules in the fluid with the colloids, thus generating the appropriate Brownian dynamics. Together, the viscous and the random-force terms in Eq. (2) represent a standard Langevin thermostat [42]. In simulations, the inter-particle potential $\phi_{\mathrm{cc}}$ is cutoff smoothly (vanishing potential and potential derivative) at a distance $R_{\text {cutoff }}=5 R_{c}$ [41].

The periodic external potential $U_{\text {ext }}(\mathbf{r})$ simulates the effects of friction against a crystalline surface. In the laboratory, this interaction has been realized by means of a modulated light field constructed by laser interference, so that its spacing and intensity can be tuned with considerable freedom [10]. In our simulations, we assume a corrugation potential of the form

$$
U_{\text {ext }}(\mathbf{r})=V_{0} W(\mathbf{r})
$$






FIG. 2. The profile $W\left(r_{x}, r_{y}\right)$ of the corrugation potential which tends to localize the colloids. It is assumed to take a periodic hexagonal-lattice form, with spacing $a_{\text {pot }}$; see Eq. (4).

Here $V_{0}$ is the amplitude of the spatial modulation and $W(\vec{r})$ is a 2D periodic function with hexagonal symmetry, period $a_{\text {pot }}$, and unit amplitude. Its explicit form is

$$
\begin{aligned}
W(\mathbf{r})= & -\frac{2}{9}\left[\frac{3}{2}+2 \cos \left(\frac{2 \pi r_{x}}{a_{\mathrm{pot}}}\right) \cos \left(\frac{2 \pi r_{y}}{\sqrt{3} a_{\mathrm{pot}}}\right)\right. \\
& \left.+\cos \left(\frac{4 \pi r_{y}}{\sqrt{3} a_{\mathrm{pot}}}\right)\right] .
\end{aligned}
$$

This function is illustrated in Fig. 2.

Following the $r_{y}=0$ path in the $x$ direction a particle goes through alternating minima and saddle points of $W(\mathbf{r})$. This is the energetically less costly trajectory that a colloid can follow when pushed by an $x$-directed force. Along this path, the external potential is a simple sinusoidal oscillation,

$$
U_{\text {ext }}\left(r_{x}, 0\right)=-V_{0}\left[\frac{5}{9}+\frac{4}{9} \cos \left(\frac{2 \pi r_{x}}{a_{\text {pot }}}\right)\right] .
$$

The resulting energy barrier separating the saddle points from the minima is therefore $\frac{8}{9} V_{0}$. In this situation the static friction of an isolated particle at $T=0$, i.e., the minimum force needed to push that particle across the potential corrugation when inertial effects are negligible, equals the largest value of $\frac{\partial}{\partial r_{x}} U_{\text {ext }}\left(r_{x}, 0\right)$, namely,

$$
F_{1 s}=\frac{8 \pi V_{0}}{9 a_{\mathrm{pot}}} .
$$

In the simulations and in the relative graphs we express all applied forces by comparison to the static friction $F_{1 s}$ of the single colloid, expressed in Eq. (6). For the lattice spacing we take $a_{\text {pot }}=1.5 R_{c}$, and select values of the particles density that favor arrays of clusters whose spacing matches precisely this separation, as discussed in Sec. IIC.

\section{The cell and the initial condition}

We simulate the 2D model in a parallelogram-shaped supercell with periodic boundary conditions (PBC), thus fixing the average density. To respect the hexagonal symmetry that the arrays repulsive colloids (or clusters thereof) tend to adopt, we use a supercell generated by two primitive vectors of equal length $L$ and forming an angle of $60^{\circ}$ with each other. For $V_{0}=0$ we are free to adopt an arbitrary cell size: in the static simulations of Sec. III we will vary $L$ freely to evaluate the energetics as a function of the colloid density and therefore determine the $T=0$ phase diagram of the free model.

For $V_{0} \neq 0$, this supercell needs to match the symmetry of the corrugation potential: we must stick to $L$ given by an integer multiple of $a_{\text {pot }}$. We settle for a compromise size $L=12 a_{\text {pot }}$, small enough to guarantee simulations involving a not-too-large number of particles (and therefore manageable simulation times) but large enough for a fair averaging over fluctuations and allowing for independent and possibly asynchronous movements of individual clusters. Accordingly, the cell contains 144 corrugation minima in an area $A=\sqrt{3} L^{2} / 2$.

As discussed below, we find that a cluster-cluster spacing $1.5 R_{c}$ as optimal for favoring a well-defined $n$-cluster phase. In the present work we focus on a lattice-matched condition between the cluster-cluster spacing and the spacing $a_{\text {pot }}$ between adjacent minima of the corrugation. Therefore in all dynamical simulations of Sec. IV we settle for fixing $a_{\text {pot }}=1.5 R_{c}$, and therefore a supercell side $L=18 R_{c}$, thus generating a supercell area $A \simeq 280.6 R_{c}^{2}$. In this supercell, the potential has 144 wells, each initially hosting a single particle or a $n=2$ - to 6-particle cluster. We simulate a total $N=144 n$ particles, which thus sample the following discrete values for the average density:

$$
\rho=\frac{N}{A}=n \frac{2}{\sqrt{3} a_{\text {pot }}^{2}}=n \frac{8}{9 \sqrt{3} R_{c}^{2}} .
$$

For the initial condition, we start off with distances $\delta=$ $0.32 R_{c}$ between the colloids within each cluster suggested by the inflection of the interparticle potential, Fig. 1. This initial configuration relaxes very quickly to the equilibrium interparticle distances that depends on the state of the system. We start with a regular lattice of preformed clusters, because the alternative possibilities of starting, e.g., with the particles regularly or randomly distributed across the supercell leads to irregular patterns involving different cluster sizes, which may describe appropriately large temperature and/or phasecoexistence conditions, but surely do not represent the optimal single-type cluster phases at $T=0$.

\section{GROUND-STATE ENERGETICS FOR THE FREE MODEL}

While at finite temperature the number of colloids in each cluster fluctuates, at $T=0$, for every given density $\rho$, one expects that an hexagonal lattice of clusters with the same number $n$ of particles represents a local minimum of the total repulsive energy. We explore the density range from $\rho=0.4 R_{c}^{-2}$ to $4 R_{c}^{-2}$. In the absence of corrugation potential $\left(V_{0}=0\right)$ and driving force $(F=0)$, for every $n$-cluster and every density (i.e., every lattice spacing of the cluster crystal), we execute a full relaxation by means of a damped dynamics, for at least a total time $2000 t_{0}$, but extending the relaxation for longer time until the residual kinetic energy is safely below $10^{-8} E_{0}$. This method leads us very close to the precise ground state. Depending on $n$ and $\rho$, the system may remain trapped 


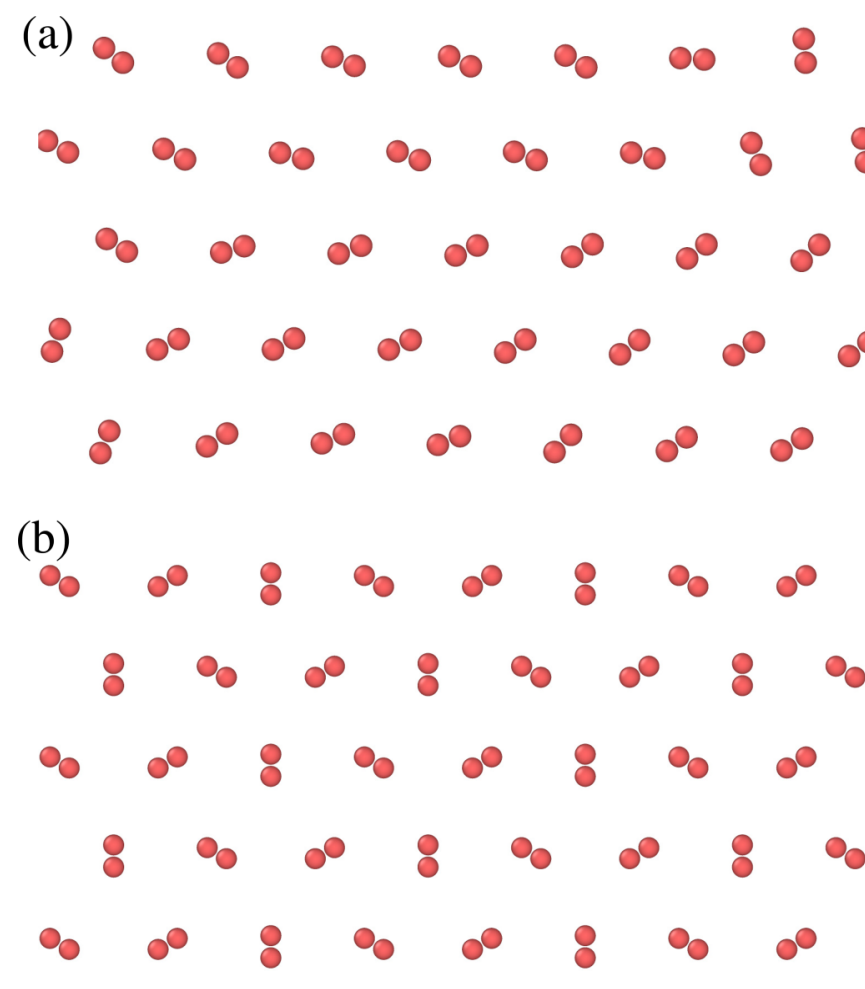

FIG. 3. Small portions of fully relaxed $T=0$ configurations representing examples of alternative local minima for the total repulsive energy of the free model $\left(V_{0}=0, F=0\right)$ in a $n=2$ cluster phase at density $\rho=16 /\left(9 \sqrt{3} R_{c}^{2}\right) \simeq 1.0264 R_{c}^{-2}$. The repulsive energy per particle of these two configurations is (a) $E=1.108486 E_{0}$; (b) $E=1.108907 E_{0}$.

in a local minimum characterized by some residual energy associated to the cluster's orientational order; see Fig. 3. We verified that these orientational-order energies are quite small, of the order of $10^{-4} E_{0}$ per particle. For this reason and for the irrelevance (discussed below) to the driven dynamics, we do not investigate the details of the orientational order in the ground state of the clustered phases.

By running several energy minimizations starting from different cluster phases, we can follow the evolution of the ground-state energy as a function of the density $\rho$. For each configuration we evaluate the total colloid-colloid repulsive energy, divide it by the number of colloids in the supercell, and obtain the energy per particle. Figure 4 displays the curves of the total energy per particle for each $n=1$ to 6 , as a function of $\rho$. We see that at each given density, several cluster configurations represent competitive local energy minima. However, at each density one of them is usually lowest in energy. Crossings between successive curves identify the coexistence density values where the $n$-cluster and the $(n+1)$-cluster have the same stability and can therefore coexist. By carrying out a finer numerical analysis and a Maxwell construction one could also identify density ranges characterized by the phase coexistence of homogeneous domains consisting of clusters of different $n$. We need not go into this detail: based on the obtained rough phase diagram, we identify the densities corresponding to stable $n$-cluster states, characterized by a cluster-cluster

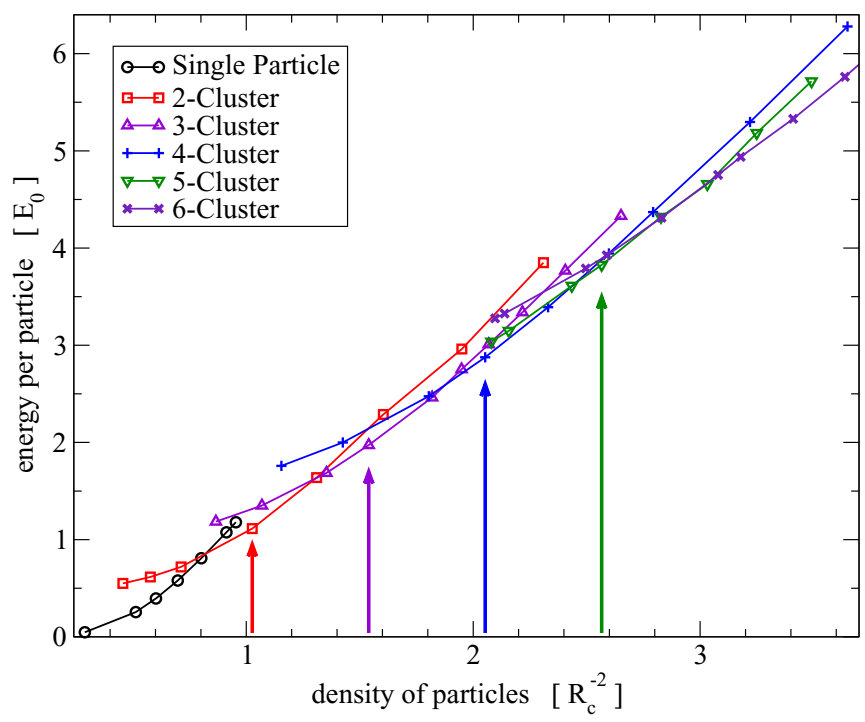

FIG. 4. The static $(T=0, F=0)$ free $\left(V_{0}=0\right)$ model total colloid-colloid repulsive energy per particle, as a function of the number density $\rho$ of colloids. Individual curves compare different $n$-cluster configurations, $n=1, \ldots 6$. The orientational order is irrelevant here, as orientational-energy differences are well within the size of the reported points; see Fig. 3. Arrows identify the densities adopted for the driven dynamics studied in Sec. IV.

spacing $1.5 R_{c}$, and we adopt them for the driven simulations of Sec. IV.

\section{DRIVEN DYNAMICS}

Starting from the equilibrated state obtained as discussed in Sec. III, we investigate the dynamics under the competing effects of the external periodic corrugation potential, which tends to immobilize the clusters at its minima, and the lateral driving force $F$ which, if there was no corrugation, would tend to establish a sliding state at speed $v=F / \eta$. As expected of a commensurate, and even lattice-matched configuration, a static pinning threshold is always present. When $F$ is slowly raised from 0 up to this pinning threshold, after an initial transient allowing for the cluster rearrangement, the steady sliding speed remains null. However, nontrivial rearrangements of the clusters are observed well in advance of depinning: the driving force clears all orientational orderings characteristic of the ground states. The clusters are forced to specific orientations, which are displayed in Fig. 5. These ferro-orientational [43,44] arrangements are made energetically advantageous by the anisotropic energy landscape present at the regions in between the minimum and the saddle point of the corrugation potential of Fig. 2, where $F$ pushes the clusters. Precisely this forceinduced ferro-orientational cluster arrangement observed for all $n$ prior to depinning [45] makes the orientational details of the free-model ground state (e.g., Fig. 3) irrelevant for the dynamics we address in the present work.

The most straightforward dynamical indicator for a driven model of this kind is the average mobility, i.e., the ratio of the average $\hat{\mathbf{x}}$-directed velocity to the driving force, which we report for different numbers $n$ of colloids per cluster. If the corrugation potential was removed $\left(V_{0}=0\right)$, then for any 




(b)

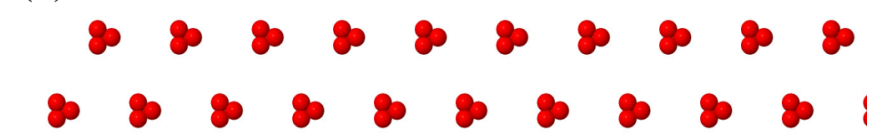

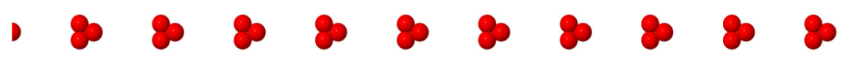

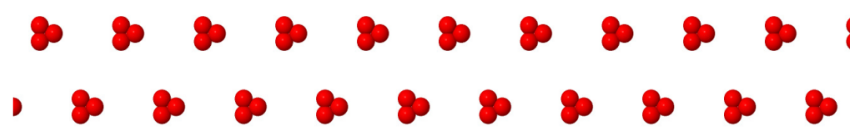

(c)



(d)

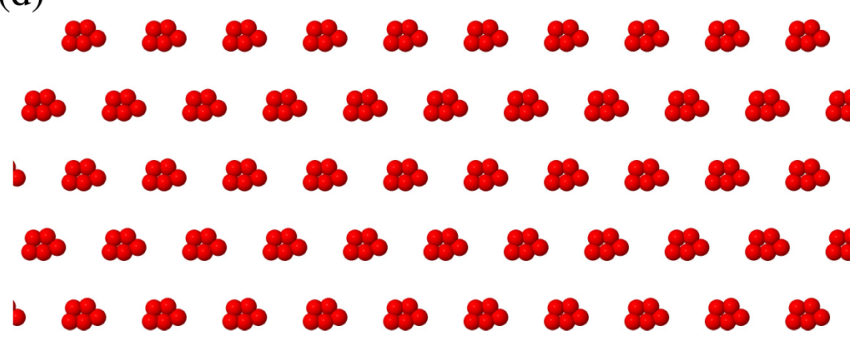

FIG. 5. Small portions of the $T=0$ configurations in a $V_{0}=1.5 E_{0}$ corrugation, under the action of a $F=0.6 F_{1 s}<F_{\text {dep }}$ pulling force, i.e., these are static configurations prior to depinning, for: (a) $n=2$; (b) $n=3$; (c) $n=4$; (d) $n=5$ clusters.

nonzero force, the mobility would equal the free mobility $\eta^{-1}$, which equals $0.0357 \eta_{0}$ with the present choice of the damping parameter. The addition of the corrugation leads to smaller values of mobility.

To evaluate the mobility, for each $2 \leqslant n \leqslant 5$ we execute three sequences of simulations, with the following amplitudes of the periodic external potential: $V_{0}=E_{0}, 1.5 E_{0}$, and $2 E_{0}$. For each sequence, we increase the applied lateral force in a range proportional to $V_{0}$, namely $0.55 F_{1 s} \leqslant F \leqslant 1.3 F_{1 s}$ in small steps $\Delta F=0.015 F_{1 s}$. At each step, the average velocity is evaluated by averaging over a total time $1400 t_{0}$ after an initial transient time $600 t_{0}$. Figures 6-9 report the resulting mobilities of the cluster lattices for the investigated

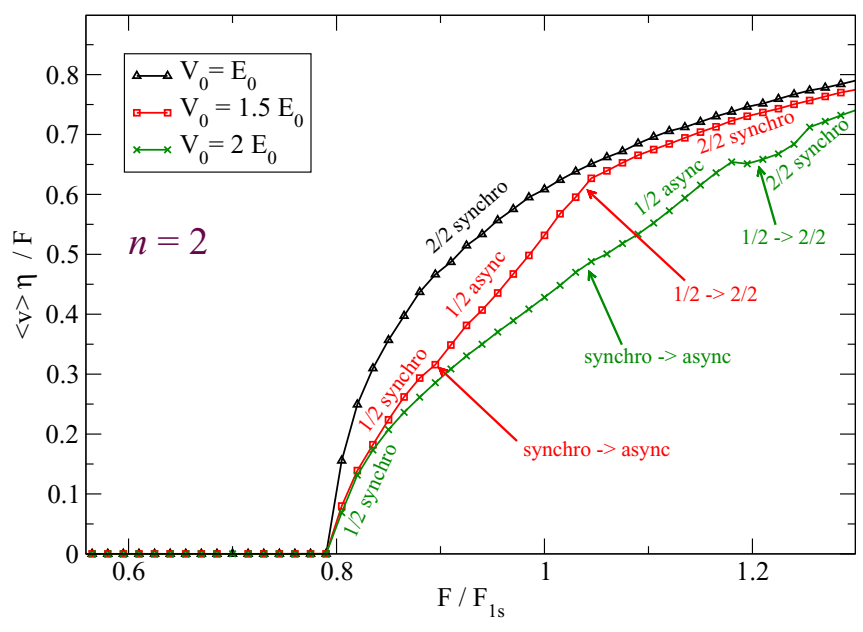

FIG. 6. The $T=0$ mobility of the $n=2$-cluster lattice as a function of the driving force $F$ expressed as a fraction of the depinning force $F_{1 s}=8 \pi V_{0} /\left(9 a_{\mathrm{pot}}\right)$ for an isolated particle, for corrugation amplitudes $V_{0} / E_{0}=1,1.5$, and 2 . The numerical error affecting the mobility due to averaging over noninteger numbers of periods is less than $0.3 \%$ of the free mobility $\eta^{-1}$. The labels along the curve identify different sliding regimes, with groups of $m / n$ particles leaving one well of $U_{\text {ext }}(\mathbf{r})$, and moving to the next well at the right. Arrows identify transitions from a sliding regime to the one occurring at larger force. Synchro/async regimes refer to whether all well-to-well particle jumps occur simultaneously across the cell or they do not. In this specific $n=2$ case, even without specification, all motions are "1D," meaning that colloids advance along horizontal lines through the minima of $W(\mathbf{r})$.

numbers $n$ of colloids per cluster as a function of the applied driving force $F$. In the initial small- $F$ simulations the driving force is insufficient to extract the colloid clusters from the potential wells of $U_{\text {ext }}$, thus a null mobility is obtained. Beyond a certain static friction threshold $F_{\text {dep }}$, the colloids unpin and start to advance, resulting in a finite mobility, which grows progressively approaching the free-particle value $\eta^{-1}$. The small "adiabatic"increase in $F$ allows us to evaluate $F_{\text {dep }}$ with a relatively small uncertainty and to identify the features of the particle motion as mobility increases after depinning.

Consider the depinning force $F_{\text {dep }}$. Although its values can be read directly from Figs. 6-9, for ease of comparison Fig. 10 reports them as a function of $n$. A first observation is that the clustering of the colloids causes a strong decrease of $F_{\text {dep }}$ compared to the value $F_{\mathrm{dep}}=F_{1 s}$ of a single colloid, by about $20 \%$ for $n=2$ and 3 up to $33 \%$ for $n=5$. The static friction force peaks for $n=3$. Above $n=3$ the depinning force tends to decrease as $n$ increases, as expected due to the growing size of the clusters.

The $n=2$ clusters mark an exception, as they depin at smaller force than $n=3$. Also, in the comparison of the depinning force for three values of $V_{0} / E_{0}$, we see that depinning for $n=2$ occurs at a smaller $F_{\text {dep }} / F_{1 s}$ for $V_{0}=E_{0}$ than $V_{0}=2 E_{0}$, contrary to all other cluster sizes. The reason for the special behavior of the $n=2$ cluster lays in its elongated conformation under traction; see Fig. 5. As a result, in the pulling direction $\hat{\mathbf{x}}$ the $n=2$ cluster extends over a longer distance $\approx \delta$ than the size $\approx \delta \sqrt{3} / 2$ of $n=3$. For this reason, when the cluster center 


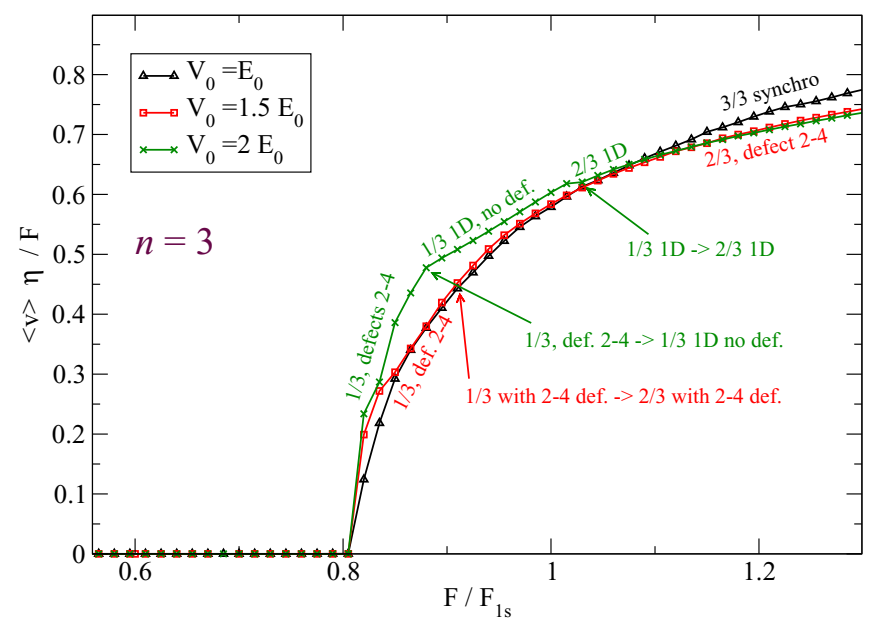

FIG. 7. Same as described in the caption of Fig. 6 but for the $n=3$ cluster lattice.

of mass approaches the inflection point of the corrugation potential (the point beyond which the retaining force begins to taper off), the rightmost particle has moved forward more for $n=2$ than for $n=3$.

After depinning, the mobility, on top of an overall smooth increase as a function of $F$, exhibits sharp changes in slope, Figs. 6-9. These changes mark transitions between different sliding patterns, which can be examined by monitoring the advancement of the individual colloids. The following section describes precisely these sliding regimes.

\section{A. Sliding regimes}

Under driving, the internal structure of the clusters makes them advance either as a single object or less trivially with structural decompositions and recompositions. Depending on the corrugation amplitude $V_{0}$ and on the number of colloids per cluster we observe (or not) several of these decompositionrecomposition phenomena as the lateral force is increased. For

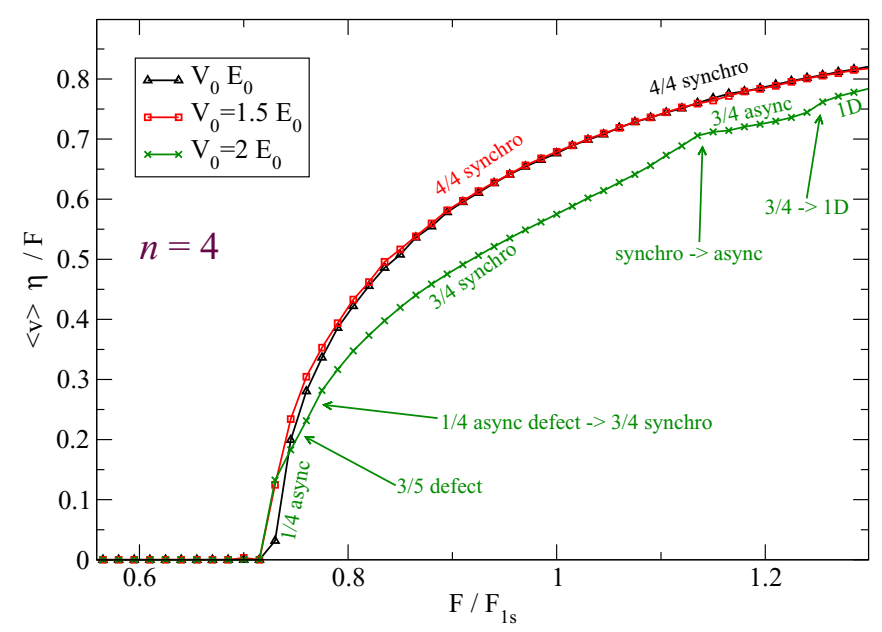

FIG. 8. Same as described in the caption of Fig. 6 but for the $n=4$ cluster lattice.

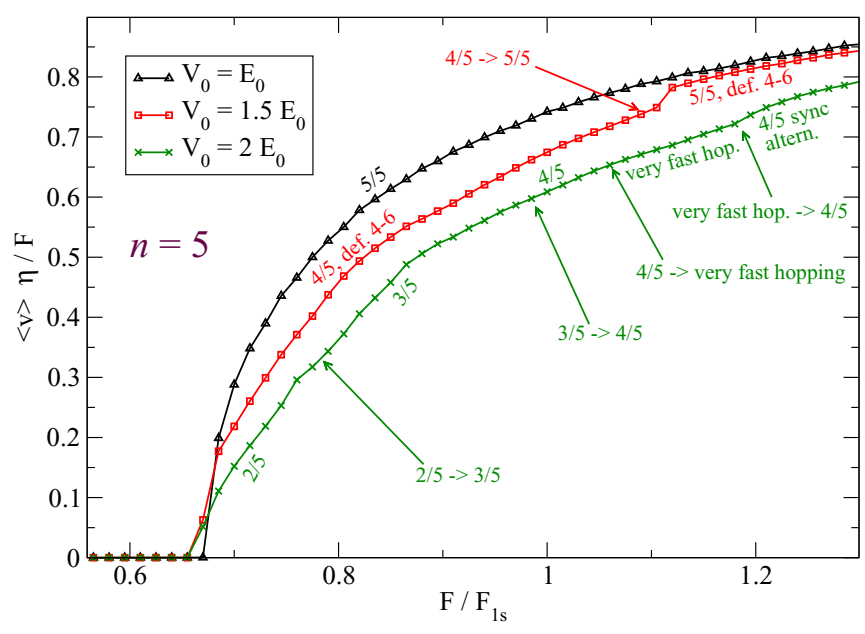

FIG. 9. Same as described in the caption of Fig. 6 but for the $n=5$ cluster lattice. All observed dynamical modes are synchronized here. See text for a description of the very fast hop and $4 / 5$ sync altern regimes.

convenience, we indicate the observed dynamical patterns on the mobility curves of Figs. 6-9, where the switching between patterns can induce visible mobility anomalies. We use $k / n$ labels indicating dynamical patterns which persist for an entire force interval. The $k / n$ labels in the mobility curves indicate that the advancement from one well to the next occurs with $k$ particles out of a cluster of $n$ jumping ahead, joining the next cluster, and leaving $n-k$ particles behind. Arrows point at transitions from one kind of dynamical behavior to the next.

As a general rule, a small corrugation amplitude $V_{0}$ tends to favor the clusters advancing as a whole, namely in " $n / n$ " modes. In contrast, for larger amplitude $V_{0}$ the

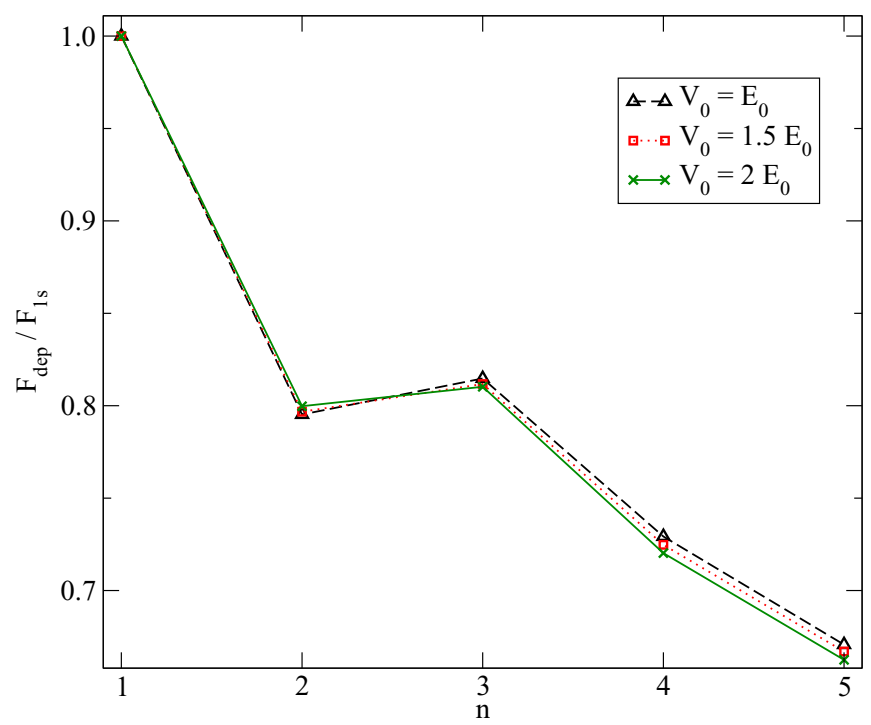

FIG. 10. The static friction, or depinning threshold, $F_{\text {dep }}$, expressed as a fraction of the single-colloid depinning force $F_{1 s}$ (also relevant for the depinning of the $n=1$ no-cluster phase), for $V_{0} / E_{0}=1$, 1.5 , and 2 , as a function of the number $n$ of colloids per cluster. 
(a)

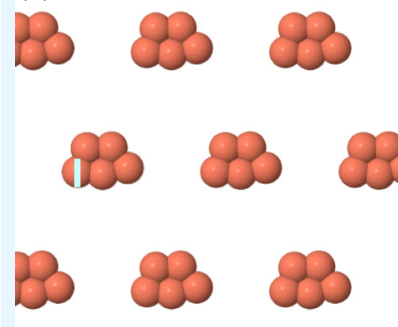

(c)

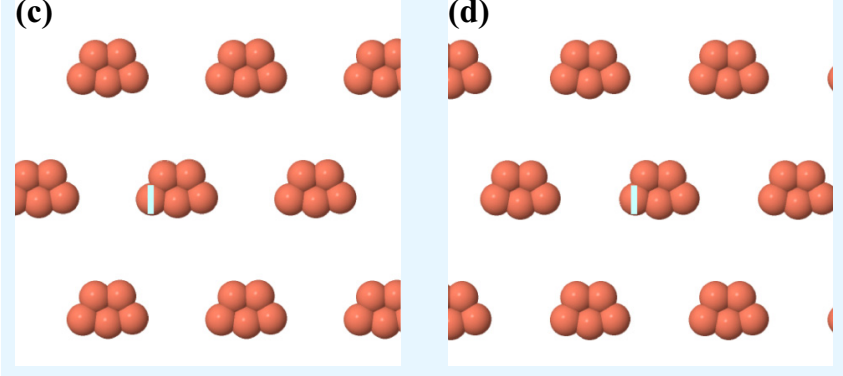

FIG. 11. The whole-cluster (5/5) advancement observed in the $n=5, V_{0}=E_{0}$ simulation driven at $F / F_{1 s}=1$; see Fig. 9. Panels (a)-(d) represent subsequent snapshots, separated by $\Delta t=2 t_{0}$. A particle is labeled with a vertical bar for ease of identification in the successive snapshots.

corrugation competes favorably with the energy advantage of the clusters to remain entire, and as a result for larger $V_{0}$ we do observe nontrivial decomposition-recomposition patterns. These decompositions can occur synchronously across the entire supercell (and in this case they are labeled synchro) or asynchronously (async label). Figures 11 and 12 report successive snapshots illustrating the two main kinds of dynamical patterns, as marked on the mobility plots. Specifically, Fig. 11 illustrates the most basic 5/5 advancement mode: whole cluster advancing from one corrugation well to the next, as observed for weak corrugation $V_{0}=E_{0}$. In contrast, Fig. 12 illustrates an advancement mode involving synchronous decompositions and recompositions of the $n=5$ clusters, with 2 particles abandoning each cluster and jumping to the next corrugation well while leaving 3 particles behind at each step. Examples of similar patterned movements involving different numbers of particles are reported in the Supplemental Material [46], as digital videos. Figure 13 illustrates the effect of these patterned movements on the $x$ coordinate of the center of mass of the colloids. As the particle hoppings are synchronized across the entire sample, the center-of-mass position $x_{\mathrm{cm}}$ exhibits relatively fast jumps during the synchronized hoppings, alternating with plateaus during the clusters rearrangements. The heights of the observed steps are a fraction of $a_{\mathrm{pot}}$, which matches precisely the specific dynamical ratio, e.g., the $2 / 5$ pattern (solid line in Fig. 13) exhibits $\frac{2}{5} a_{\text {pot }}$-high steps.

When the driving force reaches relatively large values $F \gtrsim F_{1 \mathrm{~s}}$, its action on the system can force the collective dynamics into an effectively one-dimensional (1D) dynamics, with all colloids organized in rows parallel to the direction of the driving force $(\hat{x})$. We noted this mode on the mobility plots, using the appropriate $1 D$ label, except for Fig. 6, because (a)

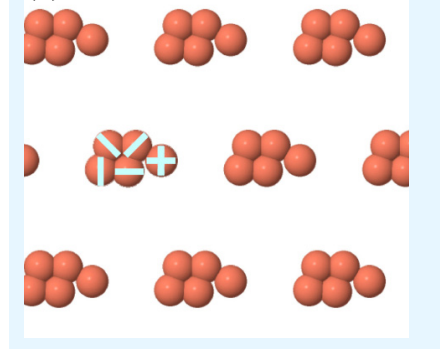

(c)

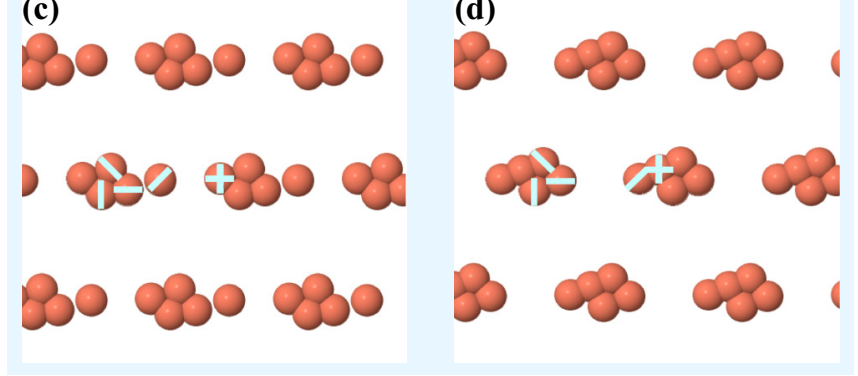

(b)

- 189808



(d)

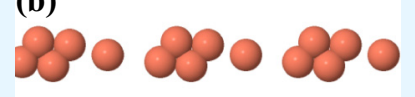

FIG. 12. Successive snapshots illustrating the $2 / 5$ dynamics observed in the $n=5, V_{0}=2 E_{0}$ simulation driven at $F / F_{1 s}=0.7$; see Fig. 9. Five particles are labeled for ease of identification in the successive snapshots. The time interval $\Delta t=2 t_{0}$, the same as described in the caption of Fig. 11.

for $n=2$ the motion is quasi-1D for any $F$. Similar quasi1D dynamics was observed also in the different model of Refs. $[37,39,45]$. In contrast for $n=5$, this quasi-1D advancement does not develop completely, because of a significant transverse ( $\hat{\mathbf{y}}$-directed) displacement persisting at all times due to the hard-core repulsion not leaving enough room for 5 colloids in a row of length $a_{\text {pot }}$. For $n=5$, the large- $F$ dynamics can consist of a rapid and uninterrupted transfer of particles from one cluster to the next, as identified by the

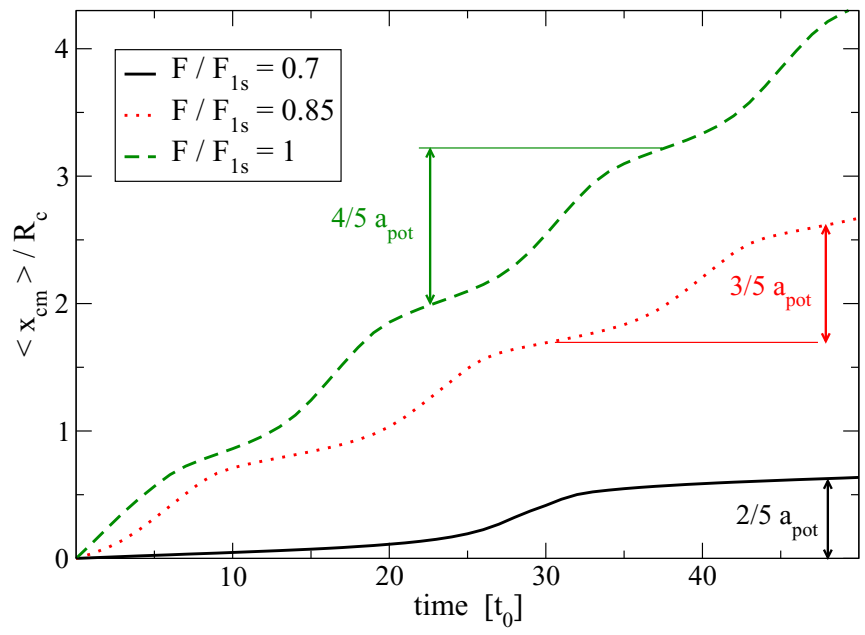

FIG. 13. The time-dependence of the $x_{\mathrm{cm}}$ of the system with $n=5$ colloids per cluster for $V_{0}=2 E_{0}$ and for three different forces representative of the fully synchronized $2 / 5,3 / 5$, and $4 / 5$ dynamical modes indicated in Fig. 9: given $a_{\text {pot }}=1.5$, the steps heights are $0.62178 \simeq \frac{2}{5} a_{\mathrm{pot}}, 0.88585 \simeq \frac{3}{5} a_{\mathrm{pot}}$, and $1.23788 \simeq \frac{4}{5} a_{\mathrm{pot}}$. 
label very fast hop in Fig. 9. Alternatively we have observed a 4/5 sync altern motion, with groups of 4 colloids jumping across clusters arranged in an alternating pattern ( 3 above +2 below)-( 2 above +3 below) along the rows, as illustrated in video cl5_4.44V2.avi in the Supplemental Material [46].

The synchronization of the well-to-well jumps can be promoted or disfavored by weak cluster-cluster interactions. As a consequence, we occasionally observe a desynchronization of the well-to-well hoppings for different clusters. In the Supplemental Material [46] we report examples of asynchronous dynamics, where the advancements at different rows occur at subsequent times. Contrasted to the synchronous advancement steps of Fig. 13, in asynchronous dynamics as a consequence of averaging no (or very small) steps are observed in the overall center-of-mass coordinate. The asynchronous jumps can occur in a regular sequence at different rows or with apparently chaotic hoppings as, for example, in the video c14_2.71V2.avi provided as Supplemental Material [46]. Note that any regularity in asynchronous motions (and possibly the overall synchronization itself when present) is favored by the specific supercell periodic boundary conditions adopted in simulation: as such, the synchronized or regular patterns may be considered artifacts of the simulated model.

This zero-temperature dynamics, often dominated by unique regular or chaotic attractors $[47,48]$, does not represent a realistic condition that one could put in relation to actual experiments. Indeed, in the more realistic finite-temperature simulations of Sec. IV B, irregular asynchronous depinning occurs in most cases, due to the Brownian forces randomly anticipating or retarding the advancement of individual particles or clusters.

By cycling the driving force $F$ up and down, we investigated whether these dynamical systems retain some kind of dynamic memory, i.e., the sliding state is affected not only by the competition of the applied confinement potential and driving but also by the initial sliding configuration. Indeed, in these $T=0$ simulations we do find some small difference of the boundaries between specific dynamical modes between the decreasing- $F$ path and the increasing- $F$ path. To rule out nonphysical underdamping effects, we verified that such hysteretic effects persist even under a doubled damping rate $\eta=$ $56 \eta_{0}$, indicating that a competition among multiple dynamical attractors is indeed present in this model.

In the present study the adopted $\hat{\mathbf{x}}$-directed orientation of the driving force tends to drive the colloids straight across the saddle points of the corrugation potential. By exploring other more general pushing directions, depending on the angle formed by the force with the $\hat{\mathbf{x}}$ direction, the colloids would be driven toward the corrugation maxima too. This kind of investigation has been carried out in the past for different models or experimental setups, typically finding locking to energetically and/or dynamically favorable advancement directions $[35,49,50]$. We expect similar directional-locking phenomena in the present model. However, possible novel regimes realized by the current cluster model may include concurrent multiple decomposition paths for the clusters, with several particles leaving simultaneously a potential well to reach different neighboring ones. We leave the investigation of these regimes to future research.

\section{B. Thermal effects}

We simulate finite temperature by restoring the random forces $\xi_{j}$ in the equations of motion Eq. (2) and therefore sampling the canonical ensemble. The starting point of the $T>0$ simulations is the appropriate $T=0$ static configuration (a perfect cluster lattice) obtained in Sec. III. In the simulated scattering intensity reported in Fig. 14(a), the modulation of the Bragg peaks reflects the structure factor of the orientationally ordered lozenge-shaped clusters at $T=0$; see Fig. 5(c). In the finite-temperature simulations of the free model $\left(V_{0}=0, F=0\right)$, it is straightforward to verify that even a low temperature, such as $T=0.01 E_{0}$ induces the melting of the orientational order of the clusters, as seen in the diffuse rings at integer multiples of $2 \pi / \delta \simeq 20 R_{c}^{-1}$ reported in Fig. 14(b). Further raising temperature leads rapidly to more disordering of the cluster crystal, and eventually to melting, which occurs between $T=0.15 E_{0}$ and $T=0.17 E_{0}$; see Figs. 14(c) and 14(d).

According to this analysis, we focus the investigation of the driven model on moderately low temperature $T \leqslant 0.05 E_{0}$. In the simulations under driving, as we did in the $T=0$ protocol, successive runs at larger and larger driving force $F$ are started from the final configuration of the previous step in $F$, which is increased "adiabatically" by $\Delta F=0.01 F_{1 s}$ at each step. For each step, the simulation duration is $500 t_{0}$ : the first $30 \%$ is dropped to prevent transient effects, and the remainder is used for the determination of the mobility, providing a fair averaging over thermal fluctuations.

Strictly speaking, at finite temperature there is no static friction, because thermal fluctuations would lead to a diffusive displacement of the layer even for $F=0$, and to a slow systematic drift in the force direction for $F>0$, if one had the patience to wait long enough for these thermally activated rare events to occur. However, at the relatively small considered temperatures $T \ll V_{0}$, the rate of well-to-well thermal-assisted hopping is extremely small, and as long as the applied force is small $F \ll F_{1 s}$, we observe no such hoppings at all for the duration of our simulations. This allows us to define $F_{\text {dep }}$ even for $T>0$, as the threshold force beyond which a significant center-of-mass displacement is observed in the applied force direction within the simulation duration. For forces immediately before this threshold, the mobility is not precisely null, but it is really tiny, with overall advancements of the entire system by far less than one lattice spacing $a_{\text {pot }}$ over the entire simulation time. We note also that all particle well-towell displacements occur within horizontal rows: although $a$ priori possible, we never observe any particle abandoning their initial $\hat{\mathbf{x}}$-oriented row and transferring to a well in the next row. These cross-row hoppings would of course become frequent for larger $T / V_{0}$. We do however observe particle jumps within rows, leading to a limited but nonzero random concentration of $n-1$ and $n+1$ "defective" clusters in the predepinning quasi-immobile state.

The mobility resulting in the finite-temperature simulations is exemplified in Fig. 15. This figure exhibits the following characteristic features, common to analogous simulations that we carried out for clusters of sizes $n=2$ to 5 , and reported in the Appendix: (i) The depinning threshold $F_{\text {dep }}$ decreases for increasing temperature, because thermal fluctuations help 

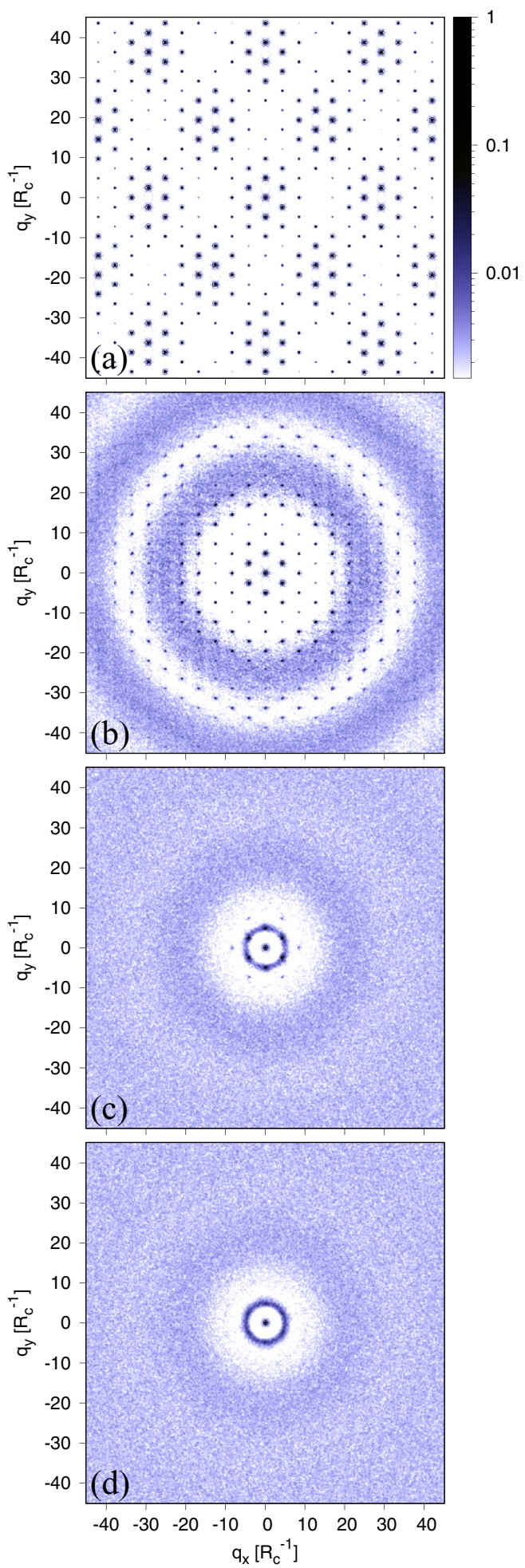

FIG. 14. The scattering intensity $S(\mathbf{q})$ obtained assuming that each colloid acts as structureless pointlike scatterer. The simulations refer to $n=4$, at four temperatures expressed in units of energy (i.e., assuming $k_{\mathrm{B}}=1$ ): (a) $T=0$-the ordered state in the static condition depicted in Fig. 5(c), (b) $T=0.01 E_{0}$-rotational melting of the cluster lattice, (c) $T=0.15 E_{0}$-crystal lattice phase just below structural melting, and (d) $T=0.17 E_{0}$-a liquid phase just above melting. The $T>0$ simulations of panels (b)-(d) are carried out for the free model $\left(V_{0}=0, F=0\right) . S(\mathbf{q})$ is averaged over 30 snapshots widely spaced in time along an equilibrium simulation.

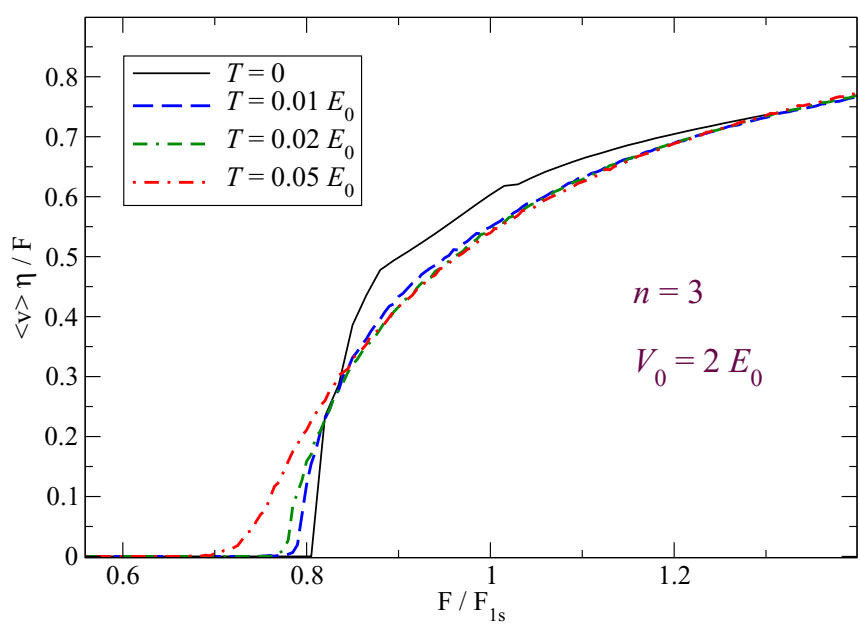

FIG. 15. The mobility, relative to the value $\eta^{-1}$ of free colloids, as a function of the driving force, relative to the $T=0$ depinning threshold $F_{1 s}$ of isolated colloids, at three relatively small but finite temperatures, expressed in units of energy $E_{0}$. The $T=0$ solid curve is the same as in Fig. 7. Simulations are carried out for $n=3$-particle clusters, for corrugation $V_{0}=2 E_{0}$. Statistical fluctuations account for error bars (not drawn) on mobility mostly smaller than $5 \times 10^{-3} \eta^{-1}$.

the particles anticipate the barrier hopping, thus activating sliding even when the driving force is nominally insufficient to overcome the barrier. (ii) For $F \gtrsim F_{\text {dep }}$, immediately above the threshold, thermal fluctuations tend to favor the sliding state, as shown by the higher mobility proving a sort of thermolubric effect [51-59]. However, depending on $n$ and the corrugation amplitude $V_{0}$, for forces larger than the $T=0$ depinning threshold $F_{1 s}$, increasing temperature can lead to either a slightly decreasing mobility, as in Fig. 15, or essentially $T$-independent mobility, quite similar to the values obtained for $T=0$; see the Appendix.

The main effect of finite temperature on the cluster dynamics is to suppress the $T=0$ synchronized advancements, especially those associated to the fractional-advancement modes. As a result, at $T>0$ the center-of-mass advancement is generally smoother, with no, or at least substantially smeared, steps like those occurring for the $T=0$ fractional sliding regimes reported in Fig. 13. Nonetheless, some amount of approximate synchronization is preserved in the special case of whole-cluster hopping, e.g., at $T>0$ the $3 / 3$ synchro pattern of Fig. 7 even extends down to smaller $F$ than for $T=0$. Relatedly, the sharp transitions between different sliding modes observed at $T=0$, at finite $T$ are replaced by smooth crossovers.

\section{DISCUSSION AND CONCLUSIONS}

In the present work we introduce and study a driven model for friction at the microscale where the protagonists are spontaneously formed clusters, rather than simple structureless objects [12,60-76] or structured but unbreakable units [77,78], as usually considered in previous investigations. We focus on a lattice-matched situation, with the external corrugation periodicity matching exactly the equilibrium spacing between the clusters. In the language of the Frenkel-Kontorova 


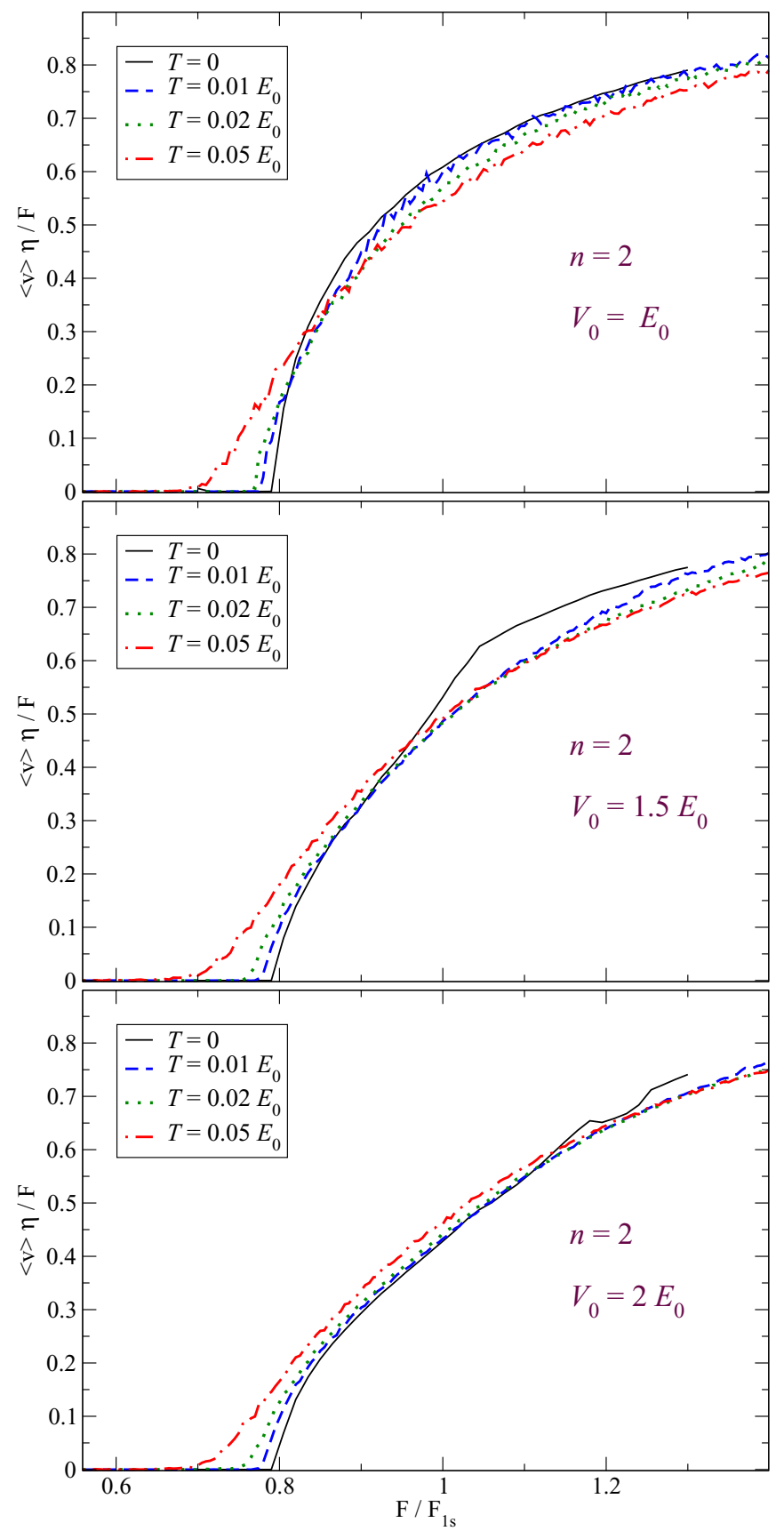

FIG. 16. The mobility $\langle v\rangle / F$, expressed in units of the free mobility $\eta^{-1}$, for clusters composed by $n=2$ particles. Simulations include $T=0$ and three finite but relatively small temperatures, expressed in units of $E_{0}$. The upper, central, and lower panels report the indicated values of the corrugation amplitude $V_{0}$, namely, $E_{0}$, $1.5 E_{0}$, and $2 E_{0}$, respectively.

model [3,79-82], this is the typical situation which maximizes the pinning effect of the corrugation, leading to a finite static friction regardless of how small the corrugation amplitude may be. We do find indeed a finite static friction in our simulations of the current model, with clustering affecting the static-friction threshold and the sliding mechanisms for driving force exceeding this threshold.

For small corrugation amplitude, the clustering tendency is dominating the dynamics, so that clusters tend to advance as a

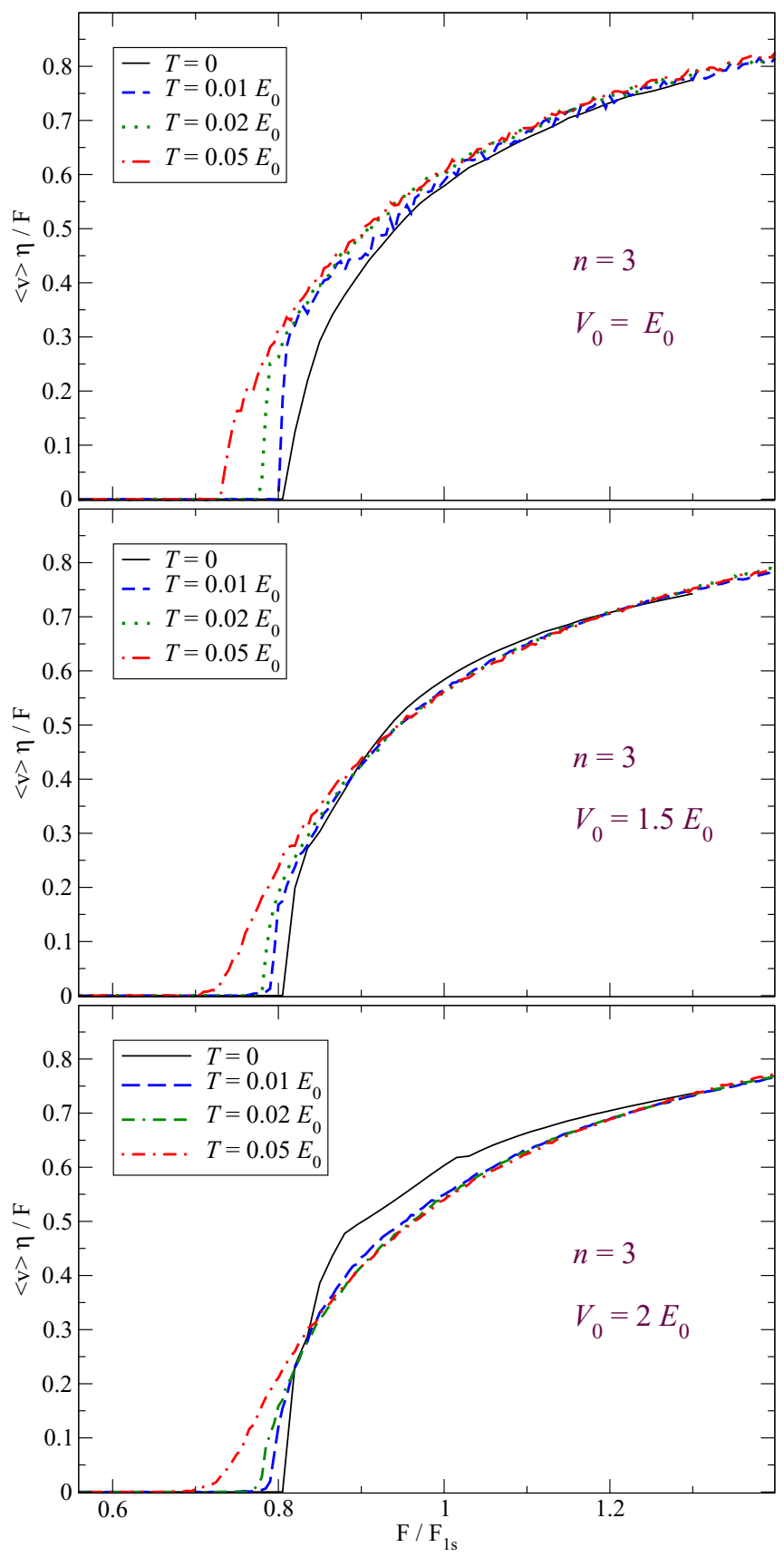

FIG. 17. Same as described in the caption of Fig. 16, but for clusters formed by $n=3$ particles.

individual objects, with the partial exception of single-particle hopping induced by thermal fluctuations near the depinning threshold. In contrast, at larger corrugation amplitude, the stability of clusters is challenged when the driving force moves them in regions where the curvature of the external corrugation potential is negative: this leads to cluster decomposition and partial hoppings. In this regime, the internal structure of the clusters decomposes in several ways as a function of the driving force, thus producing a quite rich dynamics with complicate fractional advancement patterns for clusters of various size. We investigate the impact of these phenomena on the mobility of the system. At $T=0$ we observe and 


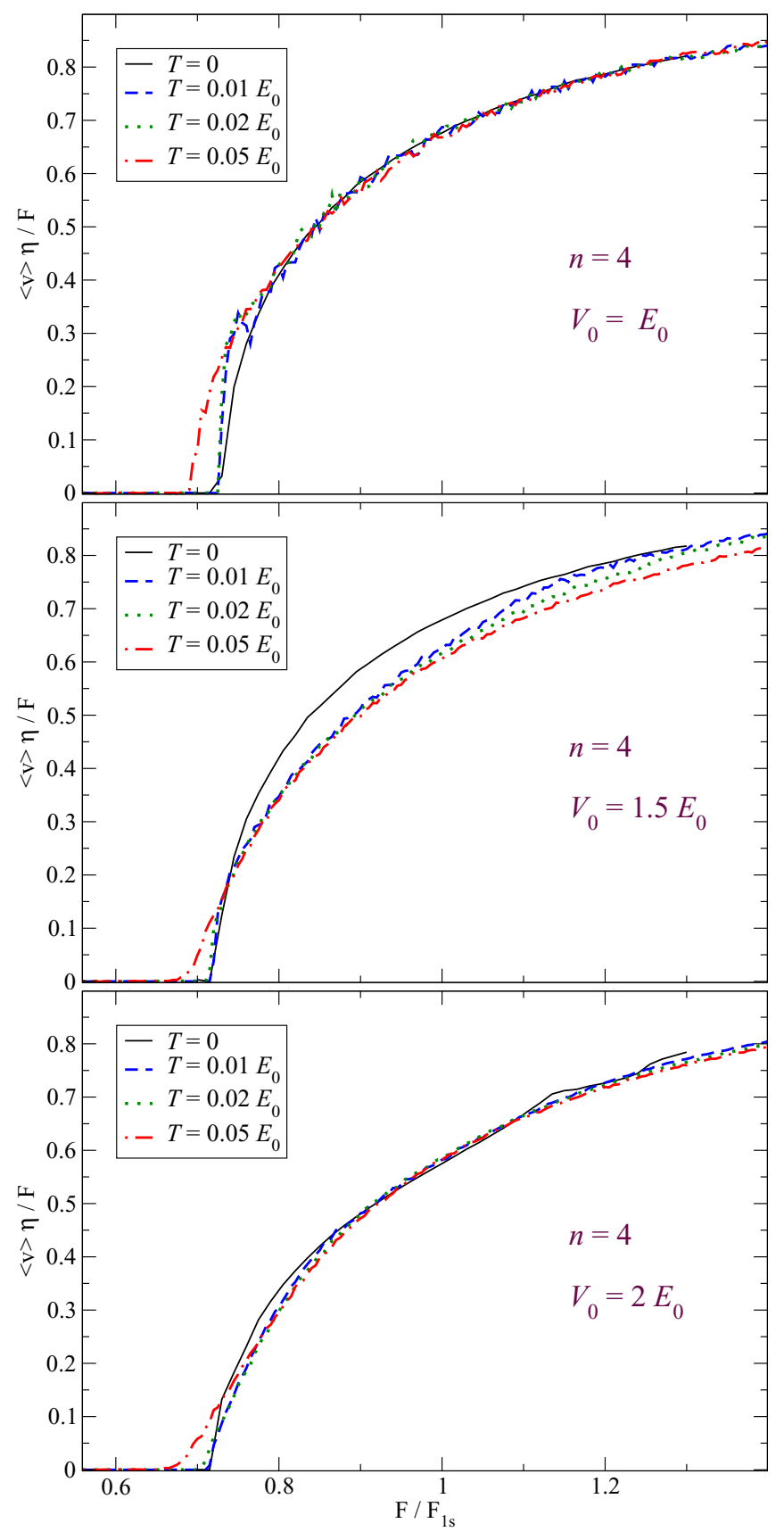

FIG. 18. Same as described in the caption of Fig. 16, but for clusters formed by $n=4$ particles.

characterize well-defined regimes of sliding of entire clusters or of decomposition-recomposition processes, with deformation of the clusters. These processes can take place in a synchronous or in asynchronous way across the system. Thermal effects tend to destroy such distinct regimes: we observe a gradual change of sliding under increasing driving force, already for temperature as low as $\sim 5 \%$ of the temperature at which the transition from a cluster crystal to a uniform fluid occurs.

The phenomenon of cluster decomposition/recomposition under driving is not expected to be specific of the interparticle interaction adopted here. We therefore predict that experiments carried out in conditions where spontaneous clustering occurs should observe this kind of dynamical behavior.

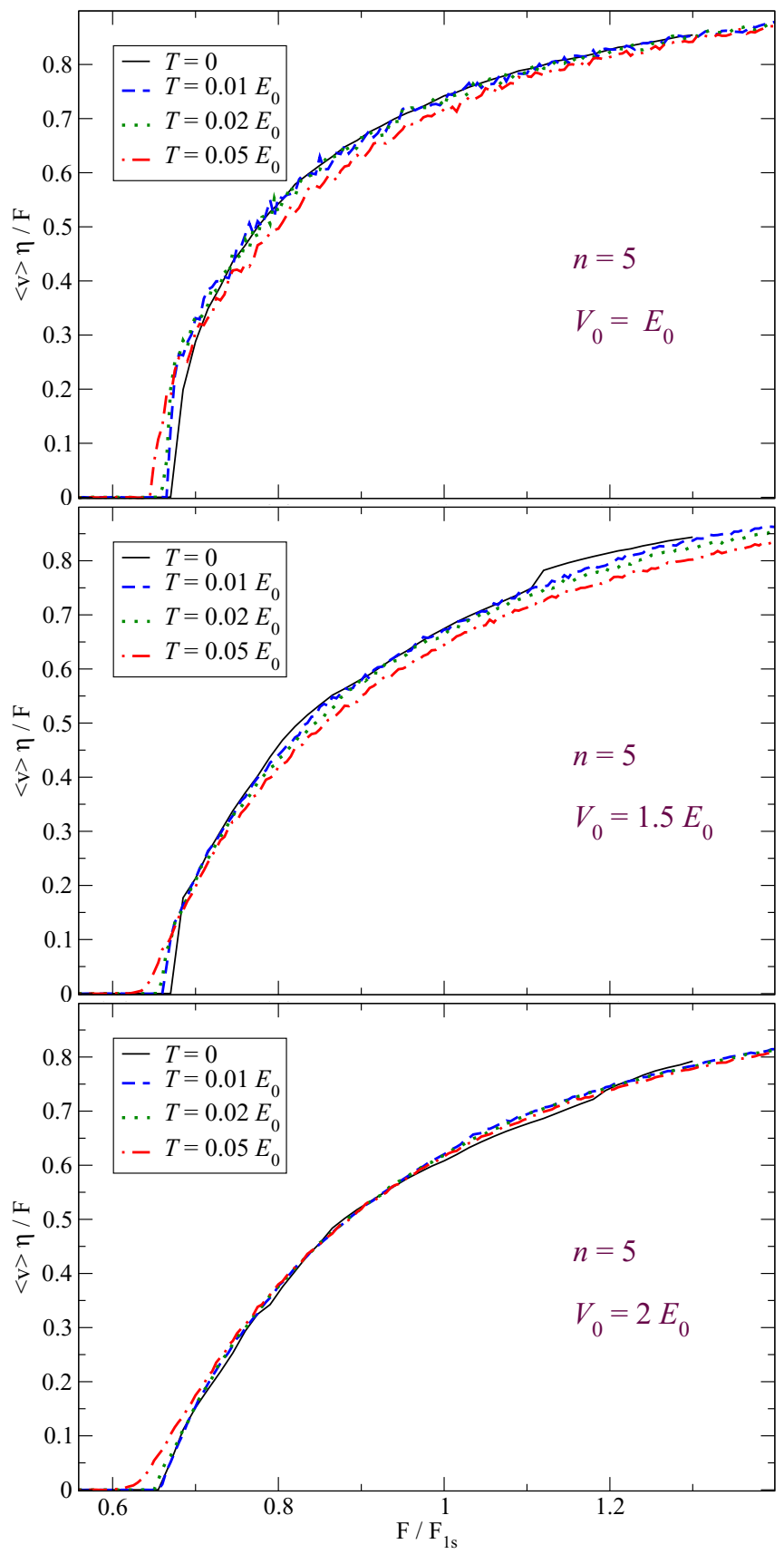

FIG. 19. Same as described in the caption of Fig. 16, but for clusters formed by $n=5$ particles.

In the future we plan to extend the investigation to latticemismatched configurations. In incommensurate conditions, for increasing corrugation amplitude $V_{0}$ there is room for an Aubry-type transition from a "superlubric" dynamics characterized by "solitonic" sliding modes [79,80,83-98]. A similar condition could be realized by taking for the substrate a symmetry other than hexagonal, as, e.g., in Refs. [72,99-101]. In the small- $V_{0}$ superlubric ground state, a finite fraction of clusters is supposed to sit near one of the maxima of the incommensurate corrugation potential, rather than all in a potential well as in the fully commensurate setup of the present paper. The novelty for a cluster-supporting condition is that, as $V_{0}$ is raised, two competing effects may arise: either an 
entire cluster near the maximum slides down toward a nearby potential well, thus leading to a relatively standard Aubry-type transition [94,97], or alternatively it decomposes under the action of divergent corrugation forces, leading to a structurally inequivalent ground state. Which of these mechanisms turns in earlier is open for investigation. These competing possibilities are likely to lead to a rich phase diagram.

\section{ACKNOWLEDGMENTS}

We acknowledge useful discussions with C. Bechinger, R. Guerra, and A. Vanossi. The COST Action MP1303 is also gratefully acknowledged.

\section{APPENDIX: THERMAL EFFECTS ON MOBILITY}

Figures 16-19 report simulated mobilities as a function of the driving force. The simulations are carried out at the values of the average particle density given by Eq. (7), for $n=2,3,4,5$, compatible with clusters of $n$ particles arranged in a regular hexagonal lattice with spacing $1.5 R_{c}$. Three different values of corrugation amplitude are considered, namely: $V_{0}=E_{0}, V_{0}=1.5 E_{0}$, and $V_{0}=2 E_{0}$. Each figure compares the $T=0$ mobility (also shown in the Figs. 6-9) with the homologous quantity obtained in finite-temperature simulations, carried out at three comparably small temperatures: $T=0.01 E_{0}, T=0.02 E_{0}$, and $T=0.05 E_{0}$.
[1] M. H. Müser, M. Urbakh, and M. O. Robbins, in Advances in Chemical Physics, Vol. 126, edited by I. Prigogine and S. A. Rice (Wiley, Hoboken, 2003), Chap. 5, p. 187.

[2] M. H. Müser, in Fundamentals of Friction and Wear on the Nanoscale, edited by E. Gnecco and E. Meyer (Springer, New York, 2007), pp. 177-199.

[3] A. Vanossi, N. Manini, M. Urbakh, S. Zapperi, and E. Tosatti, Rev. Mod. Phys. 85, 529 (2013).

[4] A. Vigentini, B. Van Hattem, E. Diato, P. Ponzellini, T. Meledina, A. Vanossi, G. Santoro, E. Tosatti, and N. Manini, Phys. Rev. B 89, 094301 (2014).

[5] N. Manini, O. M. Braun, and A. Vanossi, Nanotribology: Nonlinear mechanisms of friction, in Fundamentals of Friction and Wear on the Nanoscale, 2nd ed., edited by E. Gnecco and E. Meyer (Springer, Berlin, 2015), p. 175.

[6] N. Manini, O. M. Braun, E. Tosatti, R. Guerra, and A. Vanossi, J. Phys.: Condens. Matter 28, 293001 (2016).

[7] N. Manini, G. Mistura, G. Paolicelli, E. Tosatti, and A. Vanossi, Adv. Phys. X 2, 569 (2017).

[8] C. Apostoli, G. Giusti, J. Ciccoianni, G. Riva, R. Capozza, R. L. Woulaché, A. Vanossi, E. Panizon, and N. Manini, Beilstein J. Nanotechnol. 8, 2186 (2017).

[9] E. Panizon, G. E. Santoro, E. Tosatti, G. Riva, and N. Manini, Phys. Rev. B 97, 104104 (2018).

[10] T. Bohlein, J. Mikhael, and C. Bechinger, Nat. Mater. 11, 126 (2012).

[11] J. Hasnain, S. Jungblut, and C. Dellago, Soft Matter 9, 5867 (2013).

[12] J. Hasnain, S. Jungblut, A. Tröster, and C. Dellago, Nanoscale 6, 10161 (2014).

[13] T. Brazda, C. July, and C. Bechinger, Soft Matter 13, 4024 (2017).

[14] T. Brazda, A. Silva, N. Manini, A. Vanossi, R. Guerra, E. Tosatti, and C. Bechinger, Phys. Rev. X 8, 011050 (2018).

[15] R. Sears and W. Gelbart, J. Chem. Phys. 110, 4582 (1999).

[16] F. Sciortino, S. Mossa, E. Zaccarelli, and P. Tartaglia, Phys. Rev. Lett. 93, 055701 (2004).

[17] B. M. Mladek, D. Gottwald, G. Kahl, M. Neumann, and C. N. Likos, Phys. Rev. Lett. 96, 045701 (2006).

[18] A. J. Archer and N. B. Wilding, Phys. Rev. E 76, 031501 (2007)

[19] A. J. Archer, C. Ionescu, D. Pini, and L. Reatto, J. Phys.: Condens. Matter 20, 415106 (2008).
[20] M. Seul and D. Andelman, Science 267, 476 (1995).

[21] A. Imperio and L. Reatto, J. Phys. Condens. Matter 16, S3769 (2004).

[22] A. Imperio and L. Reatto, J. Chem. Phys. 124, 164712 (2006).

[23] R. Diaz-Mendez, F. Mezzacapo, F. Cinti, W. Lechner, and G. Pupillo, Phys. Rev. E 92, 052307 (2015).

[24] L. Leibler, Macromolecules 13, 1602 (1980).

[25] G. Fredrickson and K. Binder, J. Chem. Phys. 91, 7265 (1989).

[26] D. Pini, A. Parola, and L. Reatto, J. Chem. Phys. 143, 034902 (2015).

[27] F. Lo Verso, C. Likos, and L. Reatto, Progr. Colloid Polym. Sci. 133, 78 (2006).

[28] B. M. Mladek, G. Kahl, and C. N. Likos, Phys. Rev. Lett. 100, 028301 (2008).

[29] A. Imperio, L. Reatto, and S. Zapperi, Phys. Rev. E 78, 021402 (2008).

[30] A. Nikoubashman, G. Kahl, and C. N. Likos, Phys. Rev. Lett. 107, 068302 (2011).

[31] K. Mangold, P. Leiderer, and C. Bechinger, Phys. Rev. Lett. 90, 158302 (2003).

[32] J. Mikhael, J. Roth, L. Helden, and C. Bechinger, Nature (London) 454, 501 (2008).

[33] A. Pertsinidis and X. S. Ling, Phys. Rev. Lett. 100, 028303 (2008).

[34] J. Mikhael, M. Schmiedeberg, S. Rausch, J. Roth, H. Stark, and C. Bechinger, Proc. Natl. Acad. Sci. USA 107, 7214 (2010).

[35] T. Bohlein and C. Bechinger, Phys. Rev. Lett. 109, 058301 (2012).

[36] Q. Meng, C. N. Varney, H. Fangohr, and E. Babaev, J. Phys.: Condens. Matter 29, 035602 (2017).

[37] C. Reichhardt and C. J. O. Reichhardt, Phys. Rev. E 85, 051401 (2012).

[38] C. Reichhardt and C. J. O. Reichhardt, Phys. Rev. E 80, 022401 (2009).

[39] C. Reichhardt and C. J. O. Reichhardt, Proc. SPIE 7400, 74000J (2009).

[40] C. N. Likos, A. Lang, M. Watzlawek, and H. Löwen, Phys. Rev. E 63, 031206 (2001).

[41] L. Consonni, Meccanismi di depinning in un modello di colloidi soffici, B.Sc. thesis, University Milan, 2016.

[42] C. Gardiner, Handbook of Stochastic Methods for Physics, Chemistry and the Natural Sciences (Springer, Berlin, 1985). 
[43] Y. Ozaki, M. Prager, and B. Asmussen, J. Phys. Chem. Solids 60, 1523 (1999).

[44] A. Potočnik, N. Manini, M. Komelj, E. Tosatti, and D. Arčon, Phys. Rev. B 86, 085109 (2012).

[45] C. Reichhardt and C. J. O. Reichhardt, Phys. Rev. E 79, 061403 (2009).

[46] See Supplemental Material at http://link.aps.org/supplemental/ 10.1103/PhysRevE.97.052614 for digital videos illustrating the driven dynamics of the model.

[47] K. T. Alligood, T. D. Sauer, and J. A. Yorke, Chaos: An Introduction to Dynamical Systems (Springer, New York, 2000).

[48] E. Ott, Chaos in Dynamical Systems (Cambridge University Press, New York, 2002).

[49] C. Reichhardt and C. J. O. Reichhardt, Phys. Rev. E 69, 041405 (2004).

[50] C. Reichhardt and C. J. O. Reichhardt, Phys. Rev. Lett. 106, 060603 (2011).

[51] E. Gnecco, R. Bennewitz, T. Gyalog, C. Loppacher, M. Bammerlin, E. Meyer, and H.-J. Güntherodt, Phys. Rev. Lett. 84, 1172 (2000).

[52] Y. Sang, M. Dubé, and M. Grant, Phys. Rev. Lett. 87, 174301 (2001).

[53] O. M. Dudko, A. Filippov, J. Klafter, and M. Urbakh, Chem. Phys. Lett. 352, 499 (2002).

[54] E. Riedo, E. Gnecco, R. Bennewitz, E. Meyer, and H. Brune, Phys. Rev. Lett. 91, 084502 (2003).

[55] P. Reimann and M. Evstigneev, Phys. Rev. Lett. 93, 230802 (2004).

[56] S. Y. Krylov, K. B. Jinesh, H. Valk, M. Dienwiebel, and J. W. M. Frenken, Phys. Rev. E 71, 065101 (2005).

[57] K. B. Jinesh, S. Y. Krylov, H. Valk, M. Dienwiebel, and J. W. M. Frenken, Phys. Rev. B 78, 155440 (2008).

[58] A. Franchini, V. Bortolani, G. Santoro, and M. Brigazzi, Phys. Rev. E 78, 046107 (2008).

[59] M. Pierno, L. Bignardi, M. Righi, L. Bruschi, S. Gottardi, M. Stohr, O. Ivashenko, P. Silvestrelli, P. Rudolf, and G. Mistura, Nanoscale 6, 8062 (2014).

[60] P. Thompson and M. O. Robbins, Science 250, 792 (1990).

[61] K. Shinjo and M. Hirano, Surf. Sci. 283, 473 (1993).

[62] E. D. Smith, M. O. Robbins, and M. Cieplak, Phys. Rev. B 54, 8252 (1996).

[63] G. He, M. H. Müser, and M. O. Robbins, Science 284, 1650 (1999).

[64] M. H. Müser and M. O. Robbins, Phys. Rev. B 61, 2335 (2000).

[65] L. Consoli, H. J. F. Knops, and A. Fasolino, Phys. Rev. Lett. 85, 302 (2000).

[66] A. Vanossi, J. Röder, A. R. Bishop, and V. Bortolani, Phys. Rev. E 63, 017203 (2000).

[67] M. H. Müser, L. Wenning, and M. O. Robbins, Phys. Rev. Lett. 86, 1295 (2001).

[68] L. Consoli, H. J. F. Knops, and A. Fasolino, Phys. Rev. E 64, 016601 (2001).

[69] A. Vanossi, G. Santoro, and V. Bortolani, J. Phys.: Condens. Matter 16, S2895 (2004).

[70] R. Guerra, U. Tartaglino, A. Vanossi, and E. Tosatti, Nat. Mater. 9, 634 (2010).

[71] O. M. Braun and N. Manini, Phys. Rev. E 83, 021601 (2011).
[72] N. Manini and O. M. Braun, Phys. Lett. A 375, 2946 (2011).

[73] J. A. van den Ende, A. S. de Wijn, and A. Fasolino, J. Phys. Condens. Matter 24, 445009 (2012).

[74] N. Varini, A. Vanossi, R. Guerra, D. Mandelli, R. Capozza, and E. Tosatti, Nanoscale 7, 2093 (2015).

[75] M. Ma, A. Benassi, A. Vanossi, and M. Urbakh, Phys. Rev. Lett. 114, 055501 (2015).

[76] E. Koren and U. Duerig, Phys. Rev. B 93, 201404 (2016).

[77] O. M. Braun, N. Manini, and E. Tosatti, Phys. Rev. B 78, 195402 (2008).

[78] I. M. Sivebaek, V. N. Samoilov, and B. N. J. Persson, Phys. Rev. Lett. 108, 036102 (2012).

[79] L. Floría and J. Mazo, Adv. Phys. 45, 505 (1996).

[80] O. M. Braun and Y. Kivshar, Phys. Rep. 306, 1 (1998).

[81] O. M. Braun and Y. S. Kivshar, The Frenkel-Kontorova Model: Concepts, Methods, and Applications (Springer, Berlin, 2004).

[82] A. Popov, D. Viehman, and R. Hernandez, J. Chem. Phys. 134, 104703 (2011).

[83] S. Aubry, in Solitons and Condensed Matter Physics, edited by A. R. Bishop and T. Schneider, Springer Series in Solid State Sciences Vol. 8 (Springer, Berlin, 1978), pp. 264-277.

[84] M. Peyrard and S. Aubry, J. Phys. C: Solid State Phys. 16, 1593 (1983).

[85] G. E. Santoro, A. Vanossi, N. Manini, G. Divitini, and E. Tosatti, Surf. Sci. 600, 2726 (2006).

[86] A. Vanossi, G. E. Santoro, N. Manini, M. Cesaratto, and E. Tosatti, Surf. Sci. 601, 3670 (2007).

[87] A. Vanossi, N. Manini, F. Caruso, G. E. Santoro, and E. Tosatti, Phys. Rev. Lett. 99, 206101 (2007).

[88] N. Manini, A. Vanossi, G. E. Santoro, and E. Tosatti, Phys. Rev. E 76, 046603 (2007).

[89] M. Cesaratto, N. Manini, A. Vanossi, E. Tosatti, and G. E. Santoro, Surf. Sci. 601, 3682 (2007).

[90] I. E. Castelli, N. Manini, R. Capozza, A. Vanossi, G. E. Santoro, and E. Tosatti, J. Phys.: Condens. Matter 20, 354005 (2008).

[91] I. E. Castelli, R. Capozza, A. Vanossi, G. E. Santoro, N. Manini, and E. Tosatti, J. Chem. Phys. 131, 174711 (2009).

[92] A. Vanossi, N. Manini, and E. Tosatti, Proc. Natl. Acad. Sci. USA 109, 16429 (2012).

[93] A. Vanossi, A. Benassi, N. Varini, and E. Tosatti, Phys. Rev. B 87, 045412 (2013).

[94] D. Mandelli, A. Vanossi, N. Manini, and E. Tosatti, Phys. Rev. Lett. 114, 108302 (2015).

[95] D. Mandelli, A. Vanossi, M. Invernizzi, S. Paronuzzi, N. Manini, and E. Tosatti, Phys. Rev. B 92, 134306 (2015).

[96] S. Paronuzzi Ticco, G. Fornasier, N. Manini, G. E. Santoro, E. Tosatti, and A. Vanossi, J. Phys.: Condens. Matter 28, 134006 (2016).

[97] D. Mandelli, A. Vanossi, N. Manini, and E. Tosatti, Phys. Rev. B 95, 245403 (2017).

[98] S. Bonfanti, A. Taloni, C. Negri, A. Sellerio, N. Manini, and S. Zapperi, J. Phys. Chem. Lett. 8, 5438 (2017).

[99] B. N. J. Persson, Phys. Rev. B 48, 18140 (1993).

[100] D. McDermott, J. Amelang, C. J. O. Reichhardt, and C. Reichhardt, Phys. Rev. E 88, 062301 (2013).

[101] D. McDermott, J. Amelang, L. Lopatina, C. J. O. Reichhardt, and C. Reichhardt, Soft Matter 9, 4607 (2013). 